\title{
A New Concept for Aligning Automotive Long-Range Radar Modules Based on Wireless Microwave Sensing
}

Robert Weigel, Gabor Vinci, Benjamin Laemmle, Alexander Koelpin

Institute for Electronics Engineering, Friedrich-Alexander University of Erlangen-Nuremberg

Cauerstr. 9, 91058 Erlangen, Germany

A rising number of modern automobiles are equipped with long-, mid- or short-range radar distance sensors. Especially long-range radar based driving assistance systems operating at $77 \mathrm{GHz}$ get more and more into focus to enhance traffic safety. Especially for these long range modules the adjustment of the modules exactly in parallel to the thrust vector of the vehicle has to be done in an extremely small range of tolerances. If the misalignment angle $\alpha_{\max }$ exceeds $+/-0.5^{\circ}$ (Fig. 1) objects outside the lane at maximum detection distance $\mathrm{x}$ will be misinterpreted as being an obstacle inside the own lane. Up to now the radar beam alignment is only conducted during the vehicle's assembly in the factory. In the automobile workshops there is no possibility to measure the radiated beam. All alignment tools are based on adjusting the radar module housing by optical or other methods and rely on the exact alignment of the radar beam with respect to its housing. The achieved tolerances of such tools are quite high and cannot be verified. In this contribution a new method of aligning the radar module by directly measuring the microwave beam is presented: The six-port architecture.

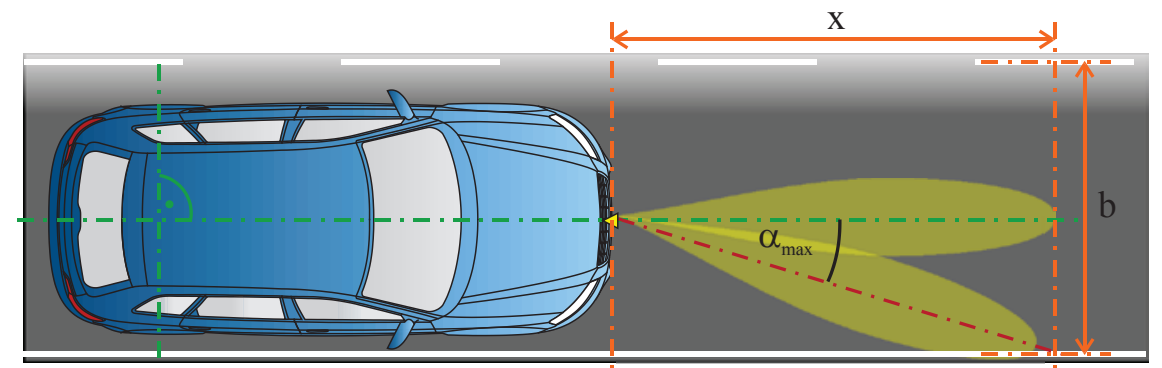

Fig. 1: Geometric dependencies for the radar module alignment in a car [6]

The six-port concept is known since mid of the 1960s for phase measurements with extremely high accuracy [1]. It has been proposed for microwave measurement setups as vector network analyzers [2]. The six-port structure generally exhibits two input ports and four output ports. The general function principle is based on the additive superposition of two microwave signals under four different relative phases. This results in constructive and destructive interference featuring in general four different microwave output signals. These signals are brought to baseband voltages form the microwave range by simply constructed diode detectors. The specific characteristics of the diodes transfer the microwave power signal to a DC voltage signal. In theses resulting voltages the original relative phase difference (and magnitude) of the two input signals is contained and can be easily reconstructed by digital signal processing. In the beginning of the six-port technique the required digital calculation complexity was a big drawback of the concept [1], but nowadays modern FPGA and DSP processors are easily able to handle the calculations.

One big advantage of the proposed six-port sensor is its low hardware complexity [3]. The real passive six-port structure is formed by transmission line microwave couplers (one Wilkinson power divider and three quadrature hybrid couplers). Despite one resistor for the power divider no additional lumped component is required. Because of its completely passive architecture a high linearity is achieved. The downconversion from the microwave range to baseband is conducted by diode detectors, constructed by a Shottky diode and a capacitor to ground. They are operated in their square law region to ensure the conversion from microwave power to baseband voltage. 


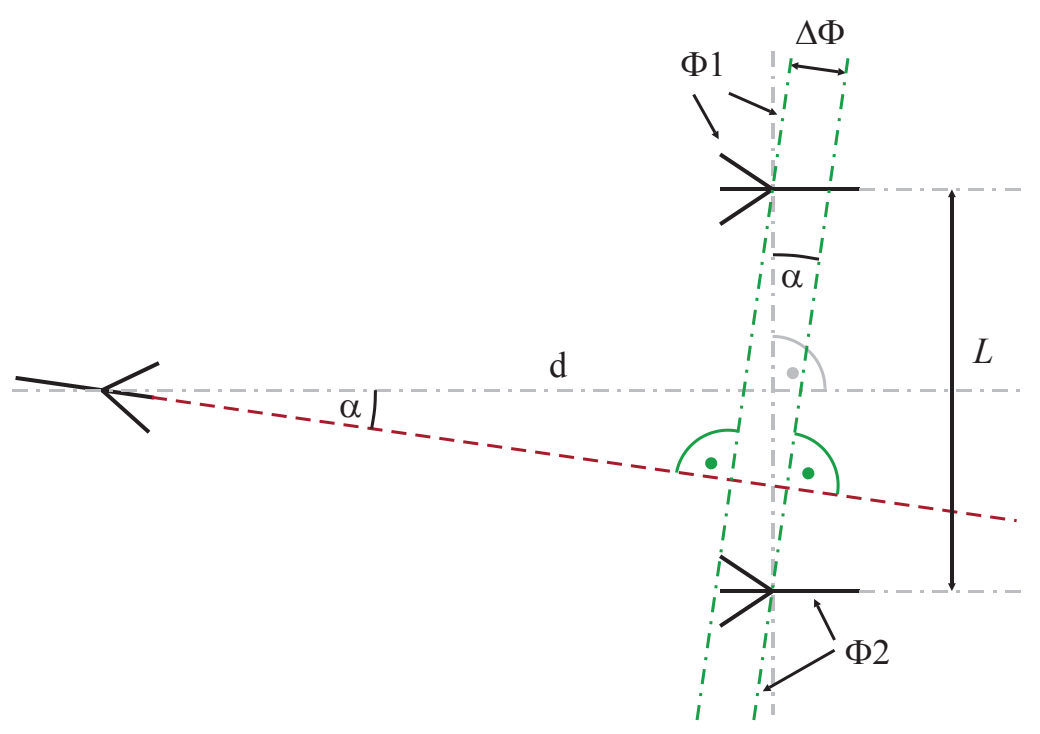

Fig. 2: Path delay $\Delta \Phi$ at antenna positions resulting from the de-adjustment angle $\alpha$ of the radar module.

The six-port concept is also a good architecture for setting up direction of arrival (DoA) measurement systems [4]. This yields from its excellent phase difference resolution. Bearing this in mind the six-port architecture is used for designing a tool for automotive radar module alignment: A microwave sensor based on DoA measurement of the radar beam. After the thrust vector $d$ of the car has been determined on a wheel alignment work station the six-port sensor is placed in front of the vehicle (Fig. 2). Two antennas are attached to the input ports of the system and the connecting line through their centers is adjusted perpendicular to the thrust vector of the car and directly in front of the radar module. The microwave beam is received by the antennas resulting in a phase difference $\Delta \Phi$ between their signals depending on the path difference as a consequence of the de-adjustment angle $\alpha$. By superimposing the two antenna signals to each other with four different relative phase shifts this path delay can be evaluated (Fig. 3) [3]. The path delay is a direct measure of the angle of arrival of the radar beam at the antenna plane. As the delay evaluation by signal superposition is done before downconverting the signals to baseband, the accuracy of the system is extremely high.

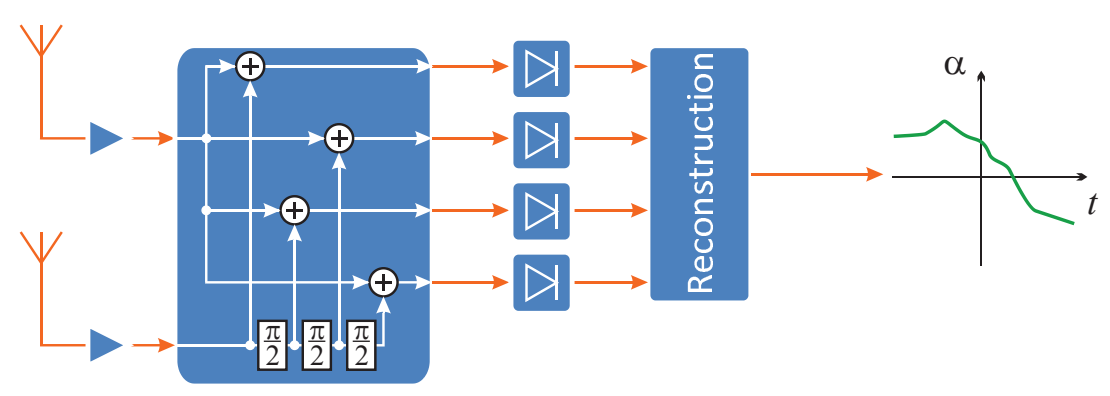

Fig. 3: The radar signal is received by two antennas. After superimposing the antennas' signals to each other with four different relative phases the resulting signals are brought to baseband by diode detectors.

The angle-of-arrival $\alpha$ can be reconstructed by the help of the four baseband voltages.

In the baseband the relative amplitudes of the four output voltages $B_{3}$ to $B_{6}$ feature the phase difference $\Delta \Phi$ between the two antenna signals (eqn. (1)).

$$
\Delta \Phi=\arctan \left(\frac{B_{3}-B_{4}}{B_{5}-B_{6}}\right)
$$

The original de-adjustment angle $\alpha$ can be reconstructed by digital signal processing solving eqn. (2).

$$
\alpha=\arcsin \left(\frac{\lambda \Delta \Phi}{2 \pi L}\right)
$$


Using this information the beam of the radar module can be adjusted manually or automatically, e.g., in a closed loop.

A six-port based DoA sensor system for aligning automotive radar modules has been built for the $24 \mathrm{GHz}$ automotive radar band (Fig. 4). The two antennas are connected to the input ports on the right and on the left followed by additional low noise amplifiers and switchable attenuators for optimum diode detector operation. In the middle of the board the transmission line couplers forming the passive six-port structure are visible. The six-port's four output signals are transferred through bandpass filters to the diodes (too small to observe in this photo) respectively. Their DC output voltages are amplified by operational amplifiers and can be tapped at the output port at the upper and the bottom edge of the board.

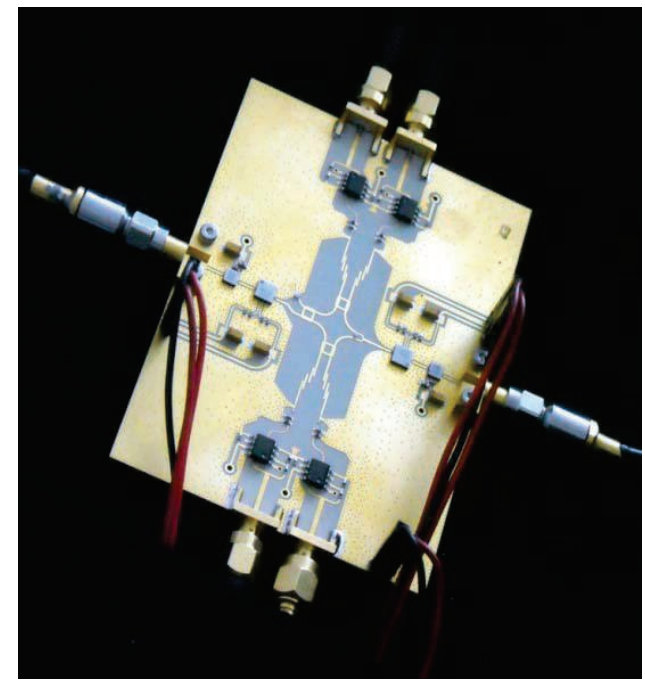

Fig. 4: Demonstrator of the complete wireless angle-of-arrival sensor. The two antennas are connected at the right and left ports; the four baseband signals are measured at the ports at the top and bottom of the circuit board. The six-port structure can be seen in the middle of the board.

The performance of the DoA sensor hardware shown in Fig. 4 has been evaluated on a test bench. The automotive radar module has been simulated by a horn antenna placed on a rotation stage 1.5 meters in front of the six-port's receiving antennas. The reconstructed alignment angle is plotted against the originally set de-adjustment angle in a range of $+/-1^{\circ}$. Because of phase periodicity an ambiguity free range between $+/-0.75^{\circ}$ can be observed showing good compliance between original and reconstructed values. An angle resolution of $0.05^{\circ}$ has been evaluated [5] even without any filtering and post processing of the measured data. The angle resolution can be further increased by this post processing. The periodicity in Fig. 5 results from the phase ambiguity in $2 \pi$.

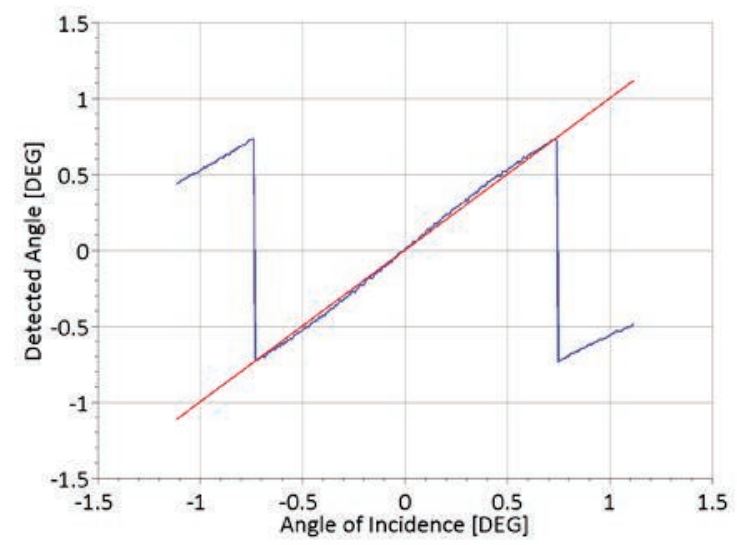

Fig. 5: Original angle of arrival versus the measured value (blue curve). The red line shows the ideal dependency.[5] 
The new concept for aligning automotive long-range radar modules based on six-port theory shows an easy to use and high accurate tool for solving this challenging task for automotive workshops. As the sixport concept is a universal wireless sensor for angle-of-arrival measurements, its application is not limited to automotive radar module alignment. It can be a useful tool for any phase difference measurement problem. Despite its extremely high accuracy there are additional advantages like low hardware complexity, low production costs and robust setup. Furthermore, the six-port based sensor can be utilized without deep knowledge of microwave theory.

\section{References:}

[1] S. B. Cohn, and N. P. Weinhouse: An automatic microwave phase measurement system, in Microwave J., vol. 7, pp. 49 - 56, Feb. 1964.

[2] G. Engen: The six-port reflectometer: An alternative network analyzer, in IEEE Transactions on Microwave Theory and Techniques, vol. 25, no. 12, pp. 1075 - 1080, December 1977.

[3] S. M. Winter, A. Koelpin, and R. Weigel: Six-port receiver analog frontend: Multilayer design and system simulation, in IEEE Transactions on Circuits and Systems II: Analog and Digital Signal Processing, vol. 55, no. 3, pp. 254 - 258, March 2008.

[4] S. O. Tatu, and T. A. Denidni: A new beam direction finding circuit based on six port technology, in Proc. IEEE MTT-S International Microwave Symposium Digest, Jun. 12.17, 2005, pp. 4

[5] G. Vinci, A. Koelpin, F. Barbon, and Robert Weigel: Six-Port-Based Direction-Of-Arrival Detection System, in. Proc. Asia-Pacific Microwave Conference, December 2010, pp.

[6] A. Kölpin: Der erweiterte Sechstor-Empfänger - Ein systemübergreifender Ansatz für Kommunikations- und Messaufgaben, dissertation (in German), Friedrich-Alexander-Universität ErlangenNürnberg, Germany, 2010 